\section{Histone deacetylase inhibitors in multiple myeloma}

\author{
Sarah Deleu,' Eline Menu,' \\ Els Van Valckenborgh, ${ }^{1}$ Ben Van Camp, ${ }^{1}$ \\ Joanna Fraczek, 'sabelle Vande Broek,' \\ Vera Rogiers, ${ }^{2}$ Karin Vanderkerken' \\ 'Myeloma Research Center-Brussels, \\ Department Hematology and \\ Immunology, Vrije Universiteit Brussel \\ (VUB), Brussels \\ ${ }^{2}$ Department of Toxicology, Vrije \\ Universiteit Brussel - VUB, Brussels, \\ Belgium
}

\begin{abstract}
Novel drugs such as bortezomib and highdose chemotherapy combined with stem cell transplantation improved the outcome of multiple myeloma patients in the past decade. However, multiple myeloma often remains incurable due to the development of drug resistance governed by the bone marrow microenvironment. Therefore targeting new pathways to overcome this resistance is needed. Histone deacetylase (HDAC) inhibitors represent a new class of anti-myeloma agents. Inhibiting HDACs results in histone hyperacetylation and alterations in chromatine structure, which, in turn, cause growth arrest differentiation and/or apoptosis in several tumor cells. Here we summarize the molecular actions of HDACi as a single agent or in combination with other drugs in different in vitro and in vivo myeloma models and in (pre-)clinical trials.
\end{abstract}

\section{Introduction}

Multiple myeloma (MM) is a plasma cell malignancy, characterized by an accumulation of monoclonal plasma cells in the bone marrow (BM) and high levels of monoclonal immunoglobulines or paraprotein in blood and/or urine. Complex interactions between MM cells and the BM microenvironment are required for the growth and progression of MM and result in the development of drug resistance, angiogenesis and induction of bone disease. ${ }^{1-4}$ Enhanced understanding of the interactions between MM and the BM microenvironment has led to the identification of new molecular targets. Novel therapeutic approaches target growth factors [e.g. insulin-like growth factor1 (IGF-1), interleukin-6 (IL-6) and vascular endothelial growth factor (VEGF)], adhesion molecules and signaling cascades in the MM cells such as the mitogen-activated protein kinase kinase (MEK)/extracellular signal regulated kinase (ERK)-pathway, the phosphatidylinositol-3 kinase (PI3K)/ protein kinase B (Akt)-pathway, the nuclear factor $\kappa$ B (NFKB)-pathway and the Wnt-pathway. ${ }^{5,6}$ Moreover, cells interacting with the MM cells in the BM, such as stromal cells, endothelial cells, osteoblasts, osteoclasts and mesenchymal stem cells are also potential targets to overcome the drug resistance against conventional chemotherapy. ${ }^{7.8}$

MM represents $1 \%$ of all cancers and it is the second most commonly diagnosed hematologic malignancy. The incidence is higher with increasing age and is 4-5 per 100,000 individuals each year worldwide. The median age at diagnosis is 67 years. ${ }^{9}$ The most common clinical characteristics in MM are bone pain, anemia, recurrent infections and renal failure. ${ }^{10}$

The standard induction therapy for elderly patients with symptomatic myeloma, and who are not candidates for stem cell transplantion, used to be melphalan (M) and prednisone (P). Recently, improved effects on survival have been seen in patients receiving MP combined with lenalidomide (Revlimid $^{\circledR}$ ) (MPR), bortezomib (Velcade ${ }^{\circledR}$ ) (MPV) or thalidomide (MPT). ${ }^{11}$ Only the latter has been accepted as standard therapy. High-dose therapy plus autologous stem cell transplantation is considered the standard therapy for front-line treatment of MM patients aged $<65$ years. ${ }^{12,13}$ The most common pre-transplantation induction therapies used today are thalidomide-dexamethasone, bortezomib-based regimes, and lenalidomide-dexamethasone. ${ }^{14,15}$ New agents such as bortezomib, thalidomide and lenalidomide in the treatment of MM do not only target the MM cells directly, but also influence the interactions of the MM cells with the BM microenvironment. Combining these new agents with conventional chemotherapy and high-dose chemotherapy with autologous stem cell transplantation increases the outcome of MM patients, although eventually all MM patients relapse. Therefore, identification of new key molecules in MM cells and in the $\mathrm{BM}$ microenvironment is crucial for the development of new therapeutic strategies.

There is growing evidence that not only gene defects such as deletions, mutations and chromosomal abnormalities are responsible for the onset and progression of cancer. Several studies have shown that epigenetic changes, i.e. heritable traits mediated by changes in DNA other than nucleotide sequences, play a key role in the downregulation of tumor suppressor genes and/or upregulation of oncogenes and, therefore, are also involved in the onset and progression of several malignancies. ${ }^{16,17}$ Chromatin remodeling is one of the main processes in epigenetic regulations. Nucleosomes are the repeating units of chromatin which contain 146 bp DNA
Correspondence: Prof. Dr. K. Vanderkerken, Department of Hematology and Immunology, Vrije Universiteit Brussel (VUB), Laarbeeklaan 103, B-1090 Brussels, Belgium

E-mail: karin.vanderkerken@vub.ac.be

Received for publication: 9 February 2009.

Revision received: 20 April 2009.

Accepted for publication: 27 April 2009.

Acknowledgment: we acknowledge grants of VUB (OZR-GOA), FWO-Vlaanderen and Stichting tegen Kanker.

This work is licensed under a Creative Commons Attribution 3.0 License (by-nc 3.0)

(C) Copyright S. Deleu et al., 2009

Licensee PAGEPress, Italy

Hematology Reviews 2009; 1:e9

doi:10.4081/hr.2009.eg

wrapped around a core histone octamer. Modifications of these nucleosomes on the histone level, as well as the DNA level, can alter the chromatin state which can be open or closed. The post-translational modifications on the core histones are most common on the amino-terminal lysine rich tail which passes through and around the enveloping DNA double helix. ${ }^{18}$ These modifications, such as acetylation, methylation, ubiquitinylation, sumoylation, phosphorylation and glycosylation are crucial in modulating gene expression, as they affect the accessibility and interaction of DNA with other non-histone protein complexes that could contain transcriptionally co-activating or co-repressing elements. ${ }^{19,20}$ Moreover, methylation of DNA, maintained by the epigenetic enzymes, methyltransferases and demethylases, also affects the chromatin structure indirectly by recruiting protein complexes containing enzymes such as histone deacetylases (HDAC).$^{21}$ HDAC and the opposite enzyme histone acetyltransferases (HAT) are the most analyzed enzymes involved in the post-translational modifications of histones. Both enzymes maintain the acetylation status of histones and non-histone proteins. HAT acetylates histones resulting in neutralizing the positive charge of histones and a more relaxed, transcriptionally active chromatin, while HDAC remove the acetyl group resulting in a more compact, transcriptionally inactive chromatin structure. ${ }^{22}$ Inhibiting HDAC leads to hyperacetylation of histones and results in gene expression alteration. In tumor cells, several HDAC inhibitors (HDACi) have shown promising anti-cancer activities with anti-proliferative, pro-apoptotic and anti-angiogeneic properties. ${ }^{23-28}$

This review provides an overview of the anti-myeloma activity of different HDACi in pre-clinical settings and the latest clinical trials with HDACi ongoing in MM patients. 


\section{The histone deacetylase family}

Eighteen HDACs have been identified in humans and are subdivided into four classes based on their homology to yeast HDACs and their enzymatic activities. ${ }^{29,30}$ Class I HDACs (1, 2,3 and 8) are homologs to the yeast $\operatorname{Rpd} 3$ and can generally be detected in the nucleus. They are ubiquitously expressed in several human cell lines and tissues. Based on phylogenetic analysis, Gregoretti et al. divided class I into Ia (HDACl and 2), Ib (HDAC3) and Ic (HDAC8) ${ }^{31}$ Class II HDACs (4, 5, 6, 7, 9 and 10) are related to yeast Hdal (histone deacetylase 1) and can shuttle between the nucleus and cytoplasm. This class is divided into class IIa (HDACs 4, 5, 7 and 9) and class IIb (HDAC6 and 10) which contain two deacetylase domains. ${ }^{30}$ Since HDAC6 contains a unique alpha-tubulin deacetylase (TCAD) domain, it can specifically deacetylate alpha-tubulin. ${ }^{32}$ The third class HDACs are the sirtuins (SIRT $1,2,3,4,5,6$ and 7) which are homologs to the yeast Sir2 (silent information regulator 2) family. These enzymes require nicotine adenine dinucleotide (NAD)+ for their deacetylase activity in contrast to the zinc-catalyzed mechanism used in class I and II HDACs. ${ }^{29}$ The sirtuins appear to deacetylate non-histone proteins and transcription factors including p53. They can not be inhibited by HDACi such as suberoylanilide hydroxamic acid (SAHA) or Trichostatin A (TSA). ${ }^{33}$ HDAC11 represents class IV and contains residues in the catalytic core regions similar to both class I and II enzymes but does not have strong enough identity to be placed in either class. ${ }^{34}$

\section{HDAC inhibitors}

Structural classification of HDAC inhibitors

Butyrate and TSA were among the first chemicals to be identified as HDAC inhibitors. Dimethylsulfoxide was used to aid superinfection of murine erythroleukemia cells with the Friend virus, whereas TSA was originally isolated as an antifungal agent from culture medium of Streptomyces hygroscopicus. Later on, it was discovered that these compounds could induce cell differentiation and a correlation with histone hyperacetylation, which was maintained by inhibiting HDACs, could be shown. ${ }^{35-39}$ It subsequently opened a new field of research. Since then, a large number of natural and synthetic HDACi have been developed by several companies and used as anti-tumor agents in pre-clinical and clinical settings (Table 1). On the basis of their chemical structure, major HDACi can be divided into four categories: short-chain fatty acids, hydroxamates, benzamides and cyclic tetrapeptides. ${ }^{26,46,47}$

Among the various classes of HDACi, short chain fatty acids such as phenylbutyrate, the anti-epileptic drug valproic acid (VPA) and sodium butyrate are only effective at mM concentrations and thereby form the less potent class of HDACi. ${ }^{41}$ Clinical evaluations have been performed with these compounds either alone or in combination and are well tolerated in patients. However due to the short plasma half-life, high doses are needed to obtain a therapeutic effect. ${ }^{40}$ The first natural hydroxamate was TSA and is now considered as the reference compound of hydroxamate based inhibitors. Most of the synthetic hydroxamate based HDACi target class I and class II with high potency. SAHA has a potency at $\mu \mathrm{M}$ range and has recently been approved for the treatment of cutaneous T-cell lymphoma. M-carboxycinamic acid bishydroxamide (CBHA) is another potent second generation inhibitor which is the structural basis for example LAQ824 and PXD101, both effective at nM range towards classes I and II. Two of the newest hydroxamate based HDACi are LBH589 and ITF2357 with very low $\mathrm{IC}_{50}$ values at $\mathrm{nM}$ concentrations. $^{43,48}$ Benzamides include MS275 and CI-994 and are generally less potent than the hydroxamates and cyclic tetrapeptides. Cyclic tetrapeptides, include the natural product depsipeptide (FK 228 or FR 901228) and apicidin. Depsipeptide is a prodrug and has to be metabolically activated via reduction of the disulfite binding. ${ }^{45}$ Recently KD5170, a novel mercaptoketone-based histone deacetylase inhibitor, has been developed. KD5170 showed significant anti-proliferative activity against a variety of human tumor cell lines, including human MM cell lines. ${ }^{44}$

Isoenzyme-selectivity of pan-HDACi and mechanism of HDAC inhibition

In general, none of these inhibitors, except tubacin, exhibit specificity towards one isoenzyme. However, they inhibit the enzyme activity of HDACs with varying efficiency (Table 1). For example, depsipeptide preferentially inhibits HDACl and 2 compared to HDAC4 and 6 , whereas the potency of MS-275 to inhibit HDAC1 is 26 times higher compared to HDAC3 and appears to lack the ability to inhibit the HDAC6 and 8. ${ }^{45,49}$ Tubacin, the HDAC6 selective inhibitor, induces hyperacetylation of $\alpha$-tubulin and has no effect on the histone acetylation status, while other hydroxamate inhibitors like TSA, SAHA and LBH589 induce histone - and $\alpha$-tubulin hyperacetylation. ${ }^{42,50-52}$

X-ray crystallographic analyses clarify the structure of an HDAC enzyme using an HDAClike protein (HDLP) isolated from an anaerobic bacterium, on the one hand and on the other hand how inhibitors such as SAHA and TSA mediate HDAC inhibition. The HDAC catalytic domain consists of a tube like pocket whereby a $\mathrm{Zn}^{2+}$ cation is positioned near the bottom of this narrow pocket. The basic structure of the HDACi contains a cap group, an aliphatic chain for a spacer, and a functional group (except depsipeptide). The cap group may be necessary for packing the inhibitor at the rim of the tube-like active site pocket, while the aliphatic group forms interactions with the residues of the lining pocket. For TSA, the hydroxamic acid group (the functional group) coordinates the zinc through its carbonyl and hydroxyl groups, resulting in the formation of a penta-coordinated zinc and thereby altering the activity of the enzyme. ${ }^{53,54}$

\section{Pre-clinical observations of HDACi in multiple myeloma}

\section{Anti-myeloma activity of HDACi as a single agent in vitro}

HDACi modulate the gene expression

profile of multiple myeloma cells

Microarray based studies showed that HDACi induce transcriptional modulations of $7-10 \%$ of the genes in malignant cell lines by acetylation of histones and non-histone proteins. ${ }^{55-57}$ The patterns of the HDACi induced gene expression alterations are quite similar for different HDAC inhibitors. Definite differences, however, could be observed by different agents in different cancer cell lines..$^{58,59}$

In MM, the first cDNA array using SAHA in the human MM1S cell line was performed by Mitsiades et al. SAHA caused selective gene expression alterations of oncogenes, proliferative/anti-apoptotic transcription factors, cell cycle regulators and members of the IGF-1R and IL-6R signaling cascades ${ }^{55}$ Recently, gene expression profiling of MM1S cells exposed to VPA have also been performed and showed that VPA also targeted genes involved in the cellular pathways crucial for the survival of the MM cells as seen for SAHA. Furthermore, they could demonstrate modulation of genes that contribute to RNA splicing/transcription and DNA replication, indicating that HDACi could affect cell growth differently from apoptosis or cell cycle regulation. ${ }^{56}$

HDACi inhibit the proliferation of multiple myeloma cells

Before investigating the molecular effect of HDACi in certain human MM cell lines, assays such as 3-(4,5-dimethylthiazol-2-yl)-2,5-diphenyl tetrazolium bromide (MTT)- or 3H-thymidine incorporation assays were performed to study the anti-proliferative effect of the HDACi. Table 2 shows an overview of different HDACi and their concentration range needed to inhibit the proliferation of the human MM cell lines and/or primary human MM cells.

HDACi such as VPA, FK228 and ITF2357 affected the viability of IL- 6 dependent as well as IL-6 independent MM cell lines, indicating 
Table 1. Isoenzyme-selectivity of pan-HDACi.

\begin{tabular}{lclll} 
Class & Compound & HDAC specificity & Company/Sponsor & Ref. \\
Short-chain fatty acid & Butyrate & Class I, Ila & Merck & 40 \\
& Valproic acid & Class I, Ila & NCI & 41 \\
Hydroxamate & SAHA & Class I, II & Merck & 40 \\
& PXD101 & Class I, II & CuraGen & 40 \\
& LAQ824 & Class I, II & Novartis & 40 \\
& LBH589 & Class I, II & Novartis & 40 \\
& Tubacin & Class IIb & BI and MIT & 42 \\
\hline Mercaptoketone & ITF2357 & Class I, II & Italfarmaco & 43 \\
Cyclic tetrapeptide & KD5170 & Class I, II & Kalypsys & 44 \\
\hline
\end{tabular}

NCI: National Cancer Institute; BI: Broad Institute; MIT: Massachusetts Institute of Technology

Table 2. Potency of HDACi used in different in vitro MM models.

\begin{tabular}{|c|c|c|c|}
\hline HDACi & Range & MM cells & Ref. \\
\hline $\mathrm{NaB}$ & $\mathrm{mM}$ & U266, RPMI 8226, ARH-77, OPM2 & 60 \\
\hline VPA & $\mathrm{mM}$ & $\begin{array}{l}\text { OPM1, MM1S, DOX-40, INA-6, OPM2, } \\
\text { NCI-H929, LP-1, RPMI 8226, U266 }\end{array}$ & $56,61,62,63$ \\
\hline SAHA & $\mu \mathrm{M}$ & MM1S & 55,64 \\
\hline LAQ824 & Sub- $\mu \mathrm{M}$ & $\begin{array}{c}\text { primary human MM cells, MM1S, } \\
\text { MM1R, RPMI 8226, -LR5, -MR20, -Dox40 }\end{array}$ & 65 \\
\hline KD5170 & $\begin{array}{c}\mathrm{R} \\
\text { Sub- } \mu \mathrm{M}\end{array}$ & $\begin{array}{c}\text { MM1S } \\
\text { H929, U266, primary human MM cells }\end{array}$ & 66 \\
\hline FK228 & $\mathrm{nM}$ & U266, RPMI 8266 & 67 \\
\hline ITF2357 & $\mathrm{nM}$ & CMA-03 & 68 \\
\hline LBH589 & $\mathrm{nM}$ & $\begin{array}{l}\text { primary human MM cells, MM1S, } \\
\text { MM1R, U266, -LR7, -Dox40 }\end{array}$ & 69 \\
\hline
\end{tabular}

MM1S: dexamethasone S, IL-6 independent; MM1R: dexamethasone R; RPMI 8226, OPM1, CMA-03, DOX-40: IL-6 independent; LR5: melphalan R; MR20: mitoxantrone R; Dox40: doxorubicin R; U266: autocrine secretion of IL-6; INA-6, CMA-03: IL-6 dependent; OPM2: IL-6 dependent, dexamethasone R when IL-6 is added; ARH-77: Epstein-Barr virus (EBV) positive cell line and thereby not considered as a genuine MM cell line..$^{70} \mathrm{~S}$ : sensitive; R: resistant

that the anti-myeloma activity of the HDACi is not influenced by one of the key growth factors in MM. ${ }^{61,67,68}$ Furthermore, co-culturing the MM cells with bone marrow stromal cells (BMSC) does not protect the cells from cell death induced by the HDACi LAQ824, ITF2357, LBH589 or KD5170. . $55,66,68,71$ These data suggest that HDACi could overcome the protective effect of the BM micro-environment. The MM1S cells were resistant to KD5170 and showed no increase in histone acetylation, whereas KD5170 sensitive cell lines exhibited histone hyperacetylation after KD5170 treatment. ${ }^{66}$ This finding indicates that inhibition of the HDAC enzymes is necessary for the antitumor effects of the HDACi.

JNJ-26481585, a recently developed novel hydroxamate based HDACi with prolonged pharmacodynamic properties, has anti-proliferative effect at $\mathrm{nM}$ concentrations in the murine 5T33MM model. ${ }^{72}$ This murine MM cell line is derived from the 5TMM mouse model which mimics the human disease closely at the molecular, cellular and clinical level. ${ }^{73,74}$
HDACi induce cell death in multiple myeloma

Besides inhibition of proliferation, HDACi induced cell death is one of the major mechanisms to inhibit the survival of the myeloma cells. Extrinsic and intrinsic apoptotic pathways as well as non-apoptotic cell death such as autophagy have been reported in myeloma cells treated with an HDACi. Figure 1 demonstrates effects of the HDACi on the compounds of the intrinsic and extrinsic apoptotic pathway.

The extrinsic apoptotic pathway is activated by ligand binding to death receptors such as Fas (Apo-1 or CD95), tumor necrosis factor receptor-1 (TNFR-1) and TNF-related apoptosis-inducing ligand (TRAIL or Apo2L) receptors (DR4 and -5), resulting in activation of caspase-8 and caspase-10. Apo2L/TRAIL interacts with two death receptors (DR4 and DR5) and potently induces apoptosis in various tumors, including primary MM cells and MM cell lines, while exerting minimal or no toxicity in normal cells. ${ }^{75,76}$

Several studies have demonstrated that HDACi can upregulate the expression of both death receptors and their ligands and are pro- posed to occur selectively in tumor cells. ${ }^{77}$ The U266 human MM cell line, although expressing significant levels of DR4 and caspase-8, is resistant to Apo2L/TRAIL and this resistance could be overcome with VPA. This sensitizing effect of VPA is mediated by the redistribution of DR4 to lipid rafts followed by an improved DR4 signaling. ${ }^{62}$ However, opposite results have been obtained by Schwartz et al. who have demonstrated that VPA activated caspase3 but not caspase- 9 and caspase- 8 in the U266, OPM2 and RPMI human MM cell lines. ${ }^{63}$ In the MM1S line, treated with LBH589, no upregulation of death receptors and their ligands could be observed. Caspase- 8 , however, was activated and the gene expression of the TOSA gene, negative regulator of the Fas ligand (FasL) or TRAIL induced apoptosis was downregulated. ${ }^{69}$ SAHA sensitized MM1S cells to a Fas-activating monoclonal antibody $\mathrm{CH}-11$ and to recombinant TRAIL. This sensitizing effect was associated with decreased expression of the antiapoptotic protein FLICE-like inhibitory protein (FLIP) and members of the inhibitors of apoptosis (IAP) family such as X-linked IAP (XIAP). ${ }^{64}$

Despite these results showing that HDACi affect the extrinsic pathway, in MM and other malignant cells it is still not clear how important the death-receptor pathway is for the therapeutic effects of HDACi.

The intrinsic apoptotic pathway is mediated by the mitochondria whereby proapoptotic signals result in the release of mitochondrial intermembrane proteins, such as cytochrome $c$ (cyto-c), apoptosis inducing factors (AIF) and second mitochondria-derived activator of caspase (Smac). Cytosolic cyto-c binds to apoptotic protease activating factor (Apaf-1), resulting in Apaf-1 oligomerization and subsequent caspase-9 activation while cytosolic Smac binds to XIAP and thereby eliminates its inhibitory effect on caspase-9. Cytosolic AIF induces caspase-independent apoptosis. ${ }^{78}$ Members from the BCL2 family partially regulating this pathway, contain the pro-apoptotic (e.g. Bax, Bak, Bid and Bim) and anti-apoptotic (e.g. Bcl2, BclxL and Mcl1) proteins. The BCL2 protein Bid, can be cleaved by caspase- 8 after deathreceptor ligation, and truncated Bid (tBid) localizes to the mitochondria to initiate the intrinsic apoptotic pathway. ${ }^{79}$

MM cells contain higher levels of the antiapoptotic proteins Bcl2 and Mcl1 and lower levels of the pro-apoptotic protein Bax compared to normal plasma cells. ${ }^{76,78}$ These findings could play a role in the survival of the MM cells and the resistance to chemotherapeutic agents.

How HDACi activate the intrinsic apoptotic cascade is cell context dependent and is still not completely understood. Treatment of the U266 MM human cell line and primary MM human cells with depsipeptide resulted in a decrease of the anti-apoptotic proteins Mcll, 


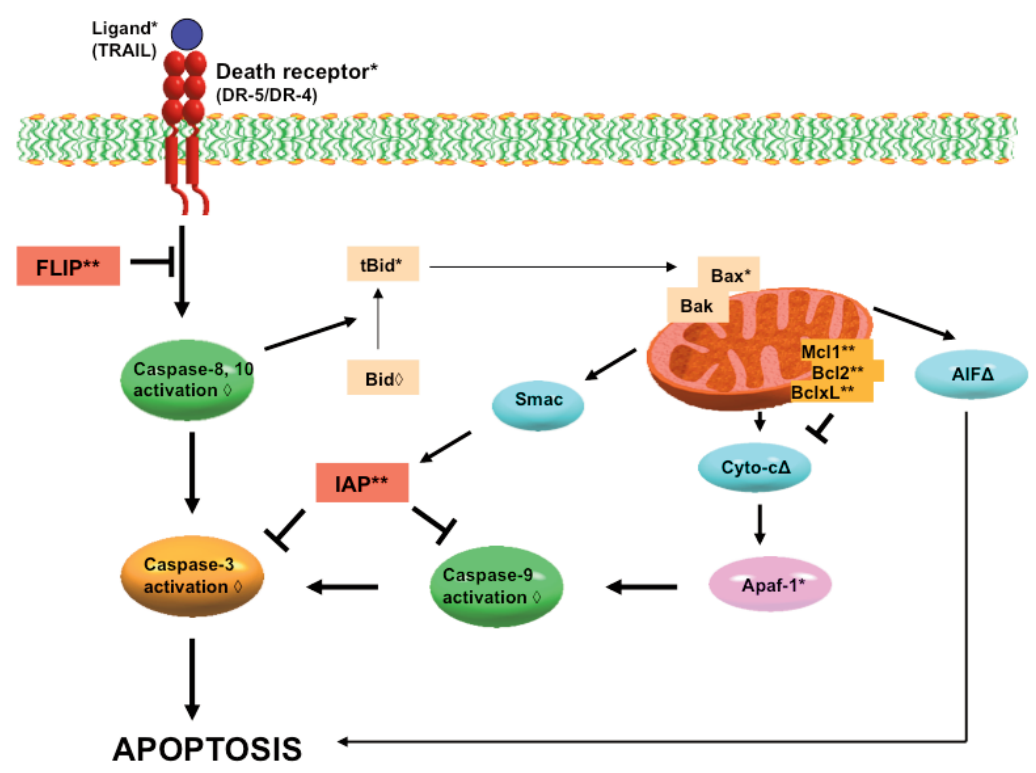

Figure 1. Induction of the extrinsic and intrinsic apoptotic pathway by HDACi in myeloma cells. The extrinsic apoptotic pathway is triggered by ligand binding and leads to activation of caspase-8, which, in turn, activates caspase-3. Activation of the intrinsic apoptotic pathway results in the release of three compounds: (a) cytochrome-c (cyto-c) which binds to apoptotic protease activating factor (Apaf-1) to activate caspase-9, (b) apoptosis inducing factors (AIF) and (c) second mitochondria-derived activator of caspase (Smac). FLICE-like inhibitory protein (FLIP) and members to the inhibitors of apoptosis (IAP) are able to prevent apoptosis induced by death receptors or intrinsic pathway respectively. Symbols denote compounds that are up-regulated $(*)$, down-regulated $\left({ }^{* *}\right)$, activated $(\diamond)$ or translocated to cytosol $(\triangle)$ by HDACi in myeloma cells.

$\mathrm{Bcl} 2, \mathrm{BclxL}$ and an increase in Bax; this latter could only be observed in primary human MM cells. $^{67}$ ITF2357 induced apoptosis through the intrinsic pathway rather than through the extrinsic pathway in the KMS18 MM cell line since no cleavage of caspase- 8 nor upregulation of DR-4 have been found, whereas cleavage of caspase- 3 and -9 and downregulation of Bcl2 and Mcl1 could be demonstrated. ${ }^{68}$ MM1S cells treated with LBH589 underwent translocation of cyto-c and AIF from the mitochondria to the cytosol, upregulation of Apaf-1 and cleavage of Bid, caspase- 9 and caspase- 3 . Furthermore, gene expression profiling revealed a novel apoptosis and caspase activation inhibitor, AVEN, which was downregulated by treatment with LBH589. ${ }^{69}$ These data represent clear evidence that LBH589 caused cell death through mitochondrial perturbations. Both LBH589 and SAHA induced poly (ADP-ribose) polymerase (PARP) cleavage in MM cells by two different enzymes, caspase- 3 and calpain, respectively. Using the calpain inhibitor, calpeptin, and the caspase-3 inhibitor, benzyloxycarbonyl-Val-Ala-Asp methylester-fluoromethylketone (z-VAD-fmk), they could demonstrate in MM1S cells that the LBH589 induced cell death is calpain-independent and partially caspase-dependent, while the SAHA induced cell death is calpain-dependent and caspaseindependent. ${ }^{64,69}$ Furthermore, SAHA promotes cleavage of Bid to tBid while overexpression of the anti-apoptotic protein Bcl2 inhibited SAHA-induced apoptotic signaling. ${ }^{64}$ Recent data indicate that KD5170 mediates cell death through mitochondrial perturbation in the U266 cells. KD5170 provoked Bax activation and cleavage of caspase- 9 and caspase-3, causing loss of mitochondrial membrane potential and subsequent pro-apoptotic factor release. The fact that AIF was released, and that the nuclear condensation was partially blocked in cells pre-treated with z-VAD-fmk before exposure to the HDACi, suggest that KD5170 induced apoptosis through both caspasedependent and caspase-independent pathways. Furthermore, KD5170 induced oxidative stress and oxidative DNA damage in myeloma cells as evidenced by the upregulation of heme oxygenase-1 and H2A.X phosphorylation, which is a marker of DNA double strand breaks. .6,80 $^{6}$

Autophagy, an alternative model for apoptosis, has been reported to contribute to the HDACi induced cell death in several tumor cell lines. ${ }^{81,82}$ Autophagy is a catabolic process involving the degradation of long-lived proteins or cytoplasmatic organelles through the lysosomal machinery. ${ }^{83}$ Schwartz et al. demonstrated for the first time that autophagy might be involved in VPA induced cytotoxicity in human myeloma cell lines. Only cleavage of caspase-3 and autophagic vacuoles in the cytoplasm could be observed in the myeloma cells treated with VPA, indicating that autophagic cell death might be involved. ${ }^{63}$

\section{HDACi induce cell cycle arrest}

HDACi, except tubacin, induce cell cycle arrest at G1/S phase. The events in the G1 phase are coordinated by the three early G1 D cyclins $(1,2,3)$ and their associated cyclindependent kinases (CDKs) 4/6 (G1 progression) and CDK 2 (G1/S transition). The transcriptional regulation of the genes, necessary for G1 progression and G1/S transition, depends on the phosphorylation state of the retinoblastoma $(\mathrm{Rb})$ protein. Phosphorylation of the Rb protein by G1 D cyclin/ cyclin-dependent kinase (CDK) results in the release of E2F, allowing transcription activation and further progression through $\mathrm{Gl}$ and initation of $\mathrm{S}$ phase. The CDK inhibitors, including the INK4 family (p16) and the Cip/Kip family (p21, p27 and $\mathrm{p57}$ ), are proteins that negatively regulate the cell cycle by competing with the cyclin D CDK binding and therefore inhibiting the CDK complex kinase activity. In MM, constitutive phosphorylation of the $\mathrm{Rb}$ protein may be fundamental to the growth and development of the tumor. $^{52}$ The mRNA level of the three G1 D cyclins are elevated in virtually all MM tumors compared to healthy plasma cells and could be due to an Ig translocation or an unknown mechanism. The elevated levels of the D cyclins are not sufficient to promote a cell cycle and need a corresponding increase of CDK4 or CDK6. ${ }^{84}$ Furthermore, several reports have demonstrated that p16 is frequently hypermethylated in primary human MM cells. However, no decreased mRNA could be found.$^{85,86}$

HDACi induce cell cycle arrest in the G1/S phase which is mostly associated with induction of p21. This has been observed in the MM cell lines treated with VPA, NVP-LAQ824,

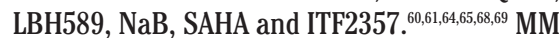
cells treated with VPA or LBH589 also showed a reduction of cyclin D1 and/or cyclin D2, indicating that induction of p21 is not solely responsible for cycle arrest. ${ }^{56,61}$

HDACi inhibit the aggresomal pathway in multiple myeloma

The aggresomal protein degradation system represents an alternative system to the proteasome for degradation of polyubquitinated misfolded/unfolded proteins (Figure 2). ${ }^{87}$ When degradation of misfolded proteins exceeds the proteasomal degradation through e.g. proteasome inhibitors, proteins interact with other unfolded or partially folded proteins, resulting 
in accumulation of ubiquitinylated proteins, organized into perinuclear stuctures termed "aggresomes" ${ }^{88,89}$ Aggresomes are formed by the retrograde transport of the aggregated proteins on microtubules (MT) and travel to the MT organizing center (MTOC) region, where they are sequestered as a single structure susceptible for lysosomal degradation. Movement of aggresomes requires intact microtubules and association with motor dynein.

HDAC6 deacetylates alfa-tubulin and plays a key role in the aggresomal pathway since it can bind polyubiquitinated proteins and dynein, facilitating the transport of aggresomes along the MTs. ${ }^{32,90}$ Targeting HDAC6 with tubacin or pan HDAC inhibitors such as SAHA or LBH589, results in hyperacetylation of alfa-tubulin, accumulation of polyubiquitinated proteins and apoptosis. ${ }^{71,91}$ It has been shown that tubacin inhibits MM cell growth in drug-sensitive (MM1S, U266, INA-6 and RPMI8226) and drug-resistant cell lines (RPMI-LR5 and RPMI-Dox40) with an $\mathrm{IC}_{50}$ between 5-20 $\mu \mathrm{M}$, whereas no cytotoxicity in peripheral blood mononuclear cells (PBMCs) could be observed at $\mu \mathrm{M}$ levels. ${ }^{92}$ This indicates that tubacin sensitivity is independent of drug resistance and that tubacin selectively targets malignant cells.

\section{HDACi affect cytokines and proteins} implicated in multiple myeloma survival, progression and immune escape

Mitsiades et al. showed that SAHA suppresses the expression of receptor genes involved in MM cell proliferation, survival and/or migration such as IGF-1R, IL-6R and its key signal transducer gp130, TNF-R, CD138 (syndecan-1) and CXCR-4. ${ }^{55}$ Furthermore, in MM1S cells they could demonstrate that SAHA suppressed autocrine IGF-1 production and paracrine IL-6 secretion of BMSC by triggering MM cell binding. This suggests that SAHA can overcome cell adhesion-mediated drug resistance. ${ }^{55,64}$ OPM- 2 cells treated with $\mathrm{NaB}$ decreased IL-6R but when cells were transfected with an expression vector of IL-6R no decrease of the receptor could be observed. Increased p21 expression and apoptosis could be observed in

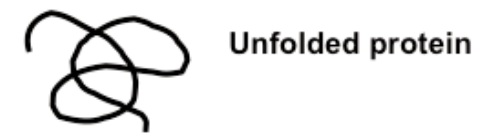

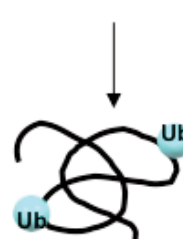

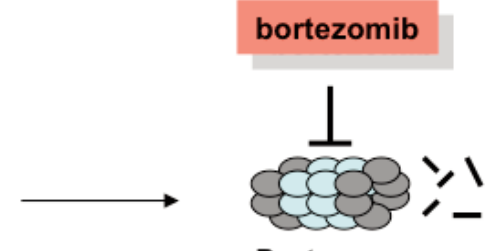

Proteasome

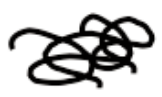

\section{Protein aggregates}
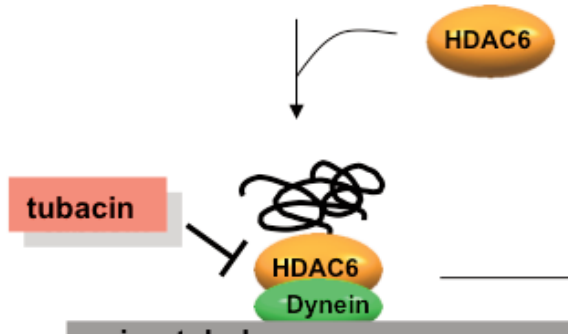

microtubule
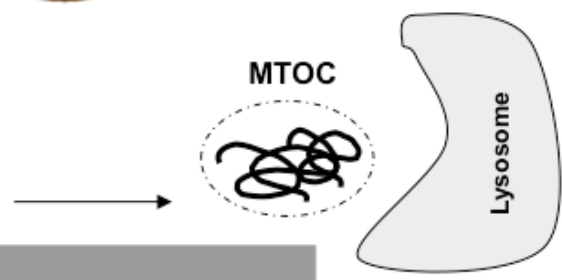

Figure 2. The aggresome pathway prevents accumulation of misfolded proteins. Unfolded or misfolded proteins, that exceed proteasomal degradation, form aggregates and are transported to the microtubule organizing center (MTOC) for degradation. This transport requires HDAC6 which deacetylates alfa-tubulin and binds both polyubiquitinated proteins and dynein. Inhibiting HDAC6 with tubacin, whether or not combined with the proteasome inhibitor bortezomib, accumulates misfolded or unfolded proteins and leads to apoptosis.

both transfected and untransfected cell lines, indicating that downregulation of the IL-6R is not required for the induction of p21 or apoptosis. ${ }^{60}$ This observation again confirms that HDACi act on multiple cellular pathways.

Several studies provide evidence that HDACi suppress angiogenesis through a direct effect on the growth and differentiation of endothelial cells on one hand and by down-regulating the expression of pro-angiogenic genes in tumor cells on the other hand. ${ }^{93-95}$ The anti-angiogenic effect of HDACi in myeloma has been demonstrated using OPM-2 and KM3 cells treated with VPA. VPA decreases VEGF secretion and VEGF receptor expression, resulting in inhibition of the vascular tubule formation of endothelial cells in co-cultures with myeloma cells. These data confirm the anti-angiogenic effect of HDACi on myeloma which is important to suppress spread of the MM cells. ${ }^{61,96,97}$

Recently, De Bruyne et al. showed that the tetraspanin CD9 which shows an inverse correlation between its expression level and tumor metastasis in solid tumors, is epigenetically down-regulated in MM and could be upregulated by treating myeloma cells with LBH589. Myeloma cells expressing CD9 become more susceptible for natural killer mediated cytolysis and the expression correlates with non-active MM disease. These observations suggest that the immune escape of the tumor cells and molecules, correlating with the MM disease status, can be affected by HDACi..$^{98}$

Anti-myeloma activity of HDACi in combination therapy in vitro

\section{Bortezomib}

Bortezomib, a first-in-class, potent and reversible proteasome inhibitor, has been successfully introduced in clinical practice and represents the standard of care in symptomatic MM patients. ${ }^{99}$ The anti-myeloma activity of bortezomib is a result of NF- $\kappa$ B inhibition, upregulation of various apoptotic pathways, and effects on the tumor micro-environment. ${ }^{100-103}$ Pei et al. were the first to demonstrate in vitro that HDACi in combination with bortezomib resulted in an improved cytotoxic effect compared to their effect as single agent. Sequential exposure of U266 and MM1S cells to bortezomib and SAHA or $\mathrm{NaB}$ potently induced caspase- $3,-8$ and -9 activation and release of the pro-apoptotic mitochondrial proteins cyto-c and Smac, resulting in a synergistic induction of apoptosis. This effect was associated with a reduction in NF- $\kappa \mathrm{B}$ DNA binding activity, modulation of JNK activation and a reactive oxygen species (ROS)-dependent downregulation of Cyclin D1, Mcl-1 and XIAP. Combining bortezomib with PXD101 caused oxidative stress accompanied by an 
enhanced effect on Bim expression, DNA damage, MAPK p38 activation and p53 phosphorylation. These observations indicate that there are several molecular mechanisms that may contribute to the synergy between bortezomib and HDACi. ${ }^{104}$ Specific inhibition of the aggresomal pathway by tubacin together with proteasome inhibition by bortezomib also resulted in an accumulation of ubiquitinated proteins followed by a synergistic anti MM-activity, mediated by stress-induced JNK activation, followed by caspase/PARP cleavage.$^{92}$ In addition, further investigations on cytoskeletal events showed that bortezomib alone lead to aggresome formation and, combining it with LBH589 or SAHA, both inhibiting HDAC6, resulted in a disruption of aggresome formation leading to apoptosis. ${ }^{71,91}$ Nawrocki et al. demonstrated that the oncogen Myc regulates the sensitivity of MM cells to bortezomib in combination with SAHA. Oncogenic activation of Myc is a hallmark of nearly all rapidly dividing malignant cells. In MM, the Myc expression is directly correlated with intracellular endoplasmatic reticulum (ER) content and protein synthesis rate. Bortezomib in combination with SAHA resulted in an induction of the pro-apoptotic BH3-only protein Noxa and ER stress indicated by a disruption of calcium homeostasis and activation of caspase-4. Further knock-down studies demonstrated that caspase- 4 and Noxa play significant roles in Myc-driven sensitivity to the combination of bortezomib and SAHA. ${ }^{91}$

Enhanced anti-MM activity of the combination therapy could not only be observed in primary human MM cells but also in co-culture conditions and conditions with exogenous growth factors IL-6 or IGF-1. Taken together, bortezomib in combination with HDACi may represent a promising therapeutic strategy that can overcome drug-resistance. ${ }^{71,69,91,92}$

\section{Death receptor ligands}

In several tumor cells, an enhanced apoptotic effect can be observed using HDACi and activators of the TRAIL and Fas pathway. However, the molecular mechanism underlying this synergism is still unclear and is celltype specific.

Fandy et al. demonstrated that TSA, as well as SAHA, in combination with TRAIL have potent synergistic effect in the AR0-1 MM cells. $^{105}$ Similar apoptotic effects have been observed in MM1S, U266 and H929 cell lines treated with KD5170 and TRAIL. ${ }^{66}$ The fact that SAHA and TSA could up-regulate the two death receptors DR4 and DR5 in the MM cells, coupled with a downregulation of anti-apoptotic proteins (Bcl-2 and XIAP) could explain the synergistic effect of combination therapy. ${ }^{105}$ However, it has been shown that HDACi could also achieve synergy with TRAIL without changing the TRAIL receptors or anti-apoptotic proteins, by simultaneously activating the intrinsic and extrinsic pathways. ${ }^{40,106}$

\section{DNA methyltransferase inhibitors}

5-azacitidine is a DNA methyltransferase inhibitor and shows activity against MM. ${ }^{107} 5$ azacitidine and analogs such as 5-azacytidine (decitabine) are interesting tools to investigate hypermethylation in tumorigenesis and the clinical efficacy is currently being assessed in phase II trials. ${ }^{108,109}$ Several investigations have already shown that hypermethylated tumor suppresor genes can be most efficiently reactivated by combining DNA demethylating agents with HDACi, this could thereby result in an enhanced reduction of tumor cell growth. . $^{110-114}$

Treatment of the human myeloma cell line, $\mathrm{U} 266$ with $\mathrm{NaB}$ and decitabine resulted in a G1 arrest, whereas no cell cycle arrest could be observed when the compounds were used as single agents. Also, the expression level of the p16 gene on RNA and protein level was significantly increased when both epigenetic agents were applied simultaneously. ${ }^{115}$ Our group could also show in the human myeloma Karpas707 cell line that the upregulation of the pro-apoptotic protein Bim by LBH589 could be enhanced by decitabine, while decitabine alone had no effect on Bim expression. ${ }^{116}$

\section{Conventional therapeutic agents}

LAQ824, depsipeptide and LBH589 showed an enhanced decrease in survival of human MM cell lines with the conventional therapeutic agents such as dexamethasone and melphalan. ${ }^{65,67,69}$ Targeting different pathways could contribute partially to the enhanced anti-MM effect; namely caspase- 8 is activated by LAQ824 and not by dexamethasone whereby combining both agents provides an additional apoptotic signal to those already induced by dexamethasone. Further investigations are needed to clarify the molecular mechanism of the synergism between chemotherapeutic agents and HDACi.

\section{Anti-myeloma activity of HDACi in vivo}

To study the pathogenesis of MM and to find new treatment strategies, different animal models have been developed, each with their own advantages and disadvantages. ${ }^{73}$

To determine whether in vivo the antimyeloma effects of LAQ824, VPA and KD5170 correlate with their in vitro activity, human MM xenografts in immunodeficient mice were used. Xenograft murine models were subcutaneously injected with RPMI8226, OPM1 or H929 and daily treatment with LAQ824, VPA or KD5170, respectively, started when tumors were measurable. These in vivo studies resulted in a significant decrease in tumor growth and a significant increase in survival of mice treated with the HDACi. ${ }^{65-67}$
Furthermore, the enhanced anti-myeloma activity of LBH589 with bortezomib could be demonstrated in vivo by Atadja et al. using a disseminated luciferized MM1S MM xenograft mouse model. ${ }^{17}$ One of the major limitations of these in vivo experiments is the lack of the interaction of MM cells with a human micro-environment and therefore a protective effect of the BM micro-environment against the anti-myeloma activity of the HDACi in vivo cannot be excluded.

Recently, the syngeneic murine 5T33 and 5T2MM models, which mimic the human myeloma disease closely, have been used to investigate the anti-myeloma activity of JNJ26481585. ${ }^{74}$ Injecting C57BV/KaLwRij mice with $5 \mathrm{~T} 2$ or $5 \mathrm{~T} 33 \mathrm{MM}$ cells results in a migration of the MM cells to the BM followed by tumor growth, induction of angiogenesis and induction of a MM bone disease (only in the 5T2MM model). 5T2 and 5T33MM mice treated with JNJ-26481585 resulted in a significant decrease in tumor load and a reduction in the MM bone disease. ${ }^{72}$ Moreover, when a very low dose of JNJ-26481585 was combined with bortezomib, MM bone disease was more reduced than seen with bortezomib alone (Deleu et al., personal observations, 2009). These in vivo studies demonstrated that the antimyeloma activity of the HDACi as single agents or in combination with bortezomib could not be overcome by the BM micro-environment.

\section{Clinical observations of HDACi in multiple myeloma}

Several clinical trials with HDACi alone or in combination with other antimyeloma agents are ongoing (Table 3$).^{118-125}$ Phase I clinical trials showed that HDACi, such as SAHA, LBH589 and depsipeptide are well tolerated in myeloma patients. In phase II clinical trials, the activity of the HDACi as single agent was limited. However, combining HDACi with dexamethasone and/or bortezomib resulted in a more promising therapeutic setting in the treatment of MM, even in patients with refractory and relapsed $\mathrm{MM}$.

\section{Future directions}

It has become clear that pan-HDACi have anti-neoplastic activities by affecting multiple pathways involved in cell growth, survival, immune response and tumor vasculature. However, the precise underlying mechanism of the inhibition of the different HDACs by panHDACi and their biological role in MM pathogenesis remain to be clarified. A greater 
Table 3. Ongoing clinical trials with HDACi as single agent or in combination therapy in MM patients.

\begin{tabular}{lcc} 
Regimen & Clinical trial & Ref. \\
Depsipeptide+bortezomib+dexamethasone & Phase I/II & 118 \\
LBH589 & Phase I/II & 119,120 \\
\hline LBH589+bortezomib & Phase IB & 121 \\
SAHA & Phase I & 122 \\
\hline SAHA+bortezomib & Phase I & 123,124 \\
ITF2357 & Phase II & 125 \\
\hline
\end{tabular}

understanding of the molecular effects of the HDACi and the role of HDACs is essential in selecting patients who are potential candidates for HDACi therapy and in designing combination studies. The development of isoformspecific inhibitors would be a valuable tool to investigate the biological role of specific HDACs. However, it is still not clear whether selective inhibition of HDACs has therapeutic advantages over a pan-HDACi. Clinical trials demonstrated promising anti-tumor responses to HDACi, mainly in combination with other agents such as bortezomib or dexamethasone which are already in clinical use. Therefore, the development of new and improved HDACi should be encouraged together with their use in combination therapy to improve the outcome for MM patients.

\section{References}

1. Atadja P, Shao W, Growney J, et al. LBH589: efficacy and protection of bone integrity in multiple myeloma as demonstrated in cell lines and in vivo mouse model. Haematologica 2007;92(s1):151.

2. Caers J, Van Valckenborgh E, Menu E, et al. Unraveling the biology of multiple myeloma disease: cancer stem cells, acquired intracellular changes and interactions with the surrounding micro-environment. Bull Cancer 2008;95:301-13.

3. Chauhan D, Anderson KC. Mechanisms of cell death and survival in multiple myeloma (MM): therapeutic implications. Apoptosis 2003;8:337-43.

4. Roodman GD. Role of the bone marrow microenvironment in multiple myeloma. $\mathrm{J}$ Bone Miner Res 2002;17:1921-5.

5. Bruno B, Giaccone L, Rotta M, Anderson K, Boccadoro M. Novel targeted drugs for the treatment of multiple myeloma: from bench to bedside. Leukemia 2005;19:172938 .

6. Piazza FA, Gurrieri C, Trentin L, Semenzato G. Towards a new age in the treat- ment of multiple myeloma. Ann Hematol 2007;86:159-72.

7. Anderson KC. Targeted therapy of multiple myeloma based upon tumor-microenvironmental interactions. Exp Hematol 2007;35: 155-62.

8. Podar K, Chauhan D, Anderson KC. Bone marrow microenvironment and the identification of new targets for myeloma therapy. Leukemia 2009;23:10-24.

9. Parkin DM, Bray F, Ferlay J, Pisani P. Global cancer statistics, 2002. CA Cancer J Clin 2005;55:74-108.

10. Jagannath S. Pathophysiological underpinnings of multiple myeloma progression. J Manag Care Pharm 2008;14:7-11.

11. Palumbo A, Falco P, Corradini P, et al. Melphalan, prednisone, and lenalidomide treatment for newly diagnosed myeloma: a report from the GIMEMA--Italian Multiple Myeloma Network. J Clin Oncol 2007;25: 4459-65.

12. Kumar SK, Dingli D, Dispenzieri A, et al. Impact of pretransplant therapy in patients with newly diagnosed myeloma undergoing autologous SCT. Bone Marrow Transplant 2008;41:1013-9.

13. Child JA, Morgan GJ, Davies FE, et al. High-dose chemotherapy with hematopoietic stem-cell rescue for multiple myeloma. $\mathrm{N}$ Engl $\mathrm{J}$ Med 2003;348:1875-83.

14. Rajkumar SV, Palumbo A. Management of newly diagnosed myeloma. Hematol Oncol Clin North Am 2007;21:1141-56, ix$\mathrm{x}$.

15. Harousseau JL. Induction therapy in multiple myeloma. Hematology Am Soc Hematol Educ Program 2008;2008:306-12.

16. Baylin SB, Ohm JE. Epigenetic gene silencing in cancer - a mechanism for early oncogenic pathway addiction? Nat Rev Cancer 2006;6:107-16.

17. Lund AH, van Lohuizen M. Epigenetics and cancer. Genes Dev 2004;18:2315-35.

18. Davie JR. Covalent modifications of histones: expression from chromatin templates. Curr Opin Genet Dev 1998;8:173-8.
19. Nightingale KP, O'Neill LP, Turner BM. Histone modifications: signalling receptors and potential elements of a heritable epigenetic code. Curr Opin Genet Dev 2006;16:125-36.

20. Strahl BD, Allis CD. The language of covalent histone modifications. Nature 2000; 403:41-5.

21. Fuks F. DNA methylation and histone modifications: teaming up to silence genes. Curr Opin Genet Dev 2005;15:4905.

22. Lehrmann H, Pritchard LL, Harel-Bellan A. Histone acetyltransferases and deacetylases in the control of cell proliferation and differentiation. Adv Cancer Res 2002;86: 41-65.

23. Dokmanovic M, Marks PA. Prospects: histone deacetylase inhibitors. J Cell Biochem 2005;96:293-304.

24. Minucci S, Pelicci PG. Histone deacetylase inhibitors and the promise of epigenetic (and more) treatments for cancer. Nat Rev Cancer 2006;6:38-51.

25. Timmermann S, Lehrmann H, Polesskaya A, Harel-Bellan A. Histone acetylation and disease. Cell Mol Life Sci 2001;58:728-36.

26. Vanhaecke T, Papeleu P, Elaut G, Rogiers V. Trichostatin A-like hydroxamate histone deacetylase inhibitors as therapeutic agents: toxicological point of view. Curr Med Chem 2004;11:1629-43.

27. Witt 0, Deubzer HE, Milde T, Oehme I. HDAC family: What are the cancer relevant targets? Cancer Lett 2009;277:8-21.

28. Marks PA, Jiang X. Histone deacetylase inhibitors in programmed cell death and cancer therapy. Cell Cycle 2005;4:549-51.

29. Blander G, Guarente L. The Sir2 family of protein deacetylases. Annu Rev Biochem 2004;73:417-35.

30. Marks PA, Dokmanovic M. Histone deacetylase inhibitors: discovery and development as anticancer agents. Expert 0pin Investig Drugs 2005;14:1497-511.

31. Gregoretti IV, Lee YM, Goodson HV. Molecular evolution of the histone deacetylase family: functional implications of phylogenetic analysis. J Mol Biol 2004; 338:17-31.

32. Hubbert C, Guardiola A, Shao R, et al. HDAC6 is a microtubule-associated deacetylase. Nature 2002;417:455-8.

33. Marmorstein R. Structure and chemistry of the Sir2 family of NAD+-dependent histone/protein deactylases. Biochem Soc Trans 2004;32:904-9.

34. Gao L, Cueto MA, Asselbergs F, Atadja P. Cloning and functional characterization of HDAC11, a novel member of the human histone deacetylase family. J Biol Chem 2002;277:25748-55.

35. Candido EP, Reeves R, Davie JR. Sodium butyrate inhibits histone deacetylation in 
cultured cells. Cell 1978;14:105-13.

36. Friend C, Scher W, Holland JG, Sato T. Hemoglobin synthesis in murine virusinduced leukemic cells in vitro: stimulation of erythroid differentiation by dimethyl sulfoxide. Proc Natl Acad Sci USA 1971;68:378-82.

37. Yoshida M, Hoshikawa Y, Koseki K, et al. Structural specificity for biological activity of trichostatin A, a specific inhibitor of mammalian cell cycle with potent differentiation-inducing activity in Friend leukemia cells. J Antibiot (Tokyo) 1990;43: 1101-6.

38. Yoshida M, Nomura S, Beppu T. Effects of trichostatins on differentiation of murine erythroleukemia cells. Cancer Res 1987; 47:3688-91.

39. Richon VM, Emiliani S, Verdin E, et al. A class of hybrid polar inducers of transformed cell differentiation inhibits histone deacetylases. Proc Natl Acad Sci USA 1998;95:3003-7.

40. Bolden JE, Peart MJ, Johnstone RW. Anticancer activities of histone deacetylase inhibitors. Nat Rev Drug Discov 2006; 5:769-84.

41. Kramer $\mathrm{OH}, \mathrm{Zhu}$ P, Ostendorff HP, et al. The histone deacetylase inhibitor valproic acid selectively induces proteasomal degradation of HDAC2. EMBO J 2003;22: 3411-20.

42. Haggarty SJ, Koeller KM, Wong JC, et al. Domain-selective small-molecule inhibitor of histone deacetylase 6 (HDAC6)-mediated tubulin deacetylation. Proc Natl Acad Sci USA 2003;100:4389-94.

43. Garber K. HDAC inhibitors overcome first hurdle. Nat Biotechnol 2007;25:17-9.

44. Hassig CA, Symons KT, Guo X, Nguyen PM, Annable T, Wash PL, et al. KD5170, a novel mercaptoketone-based histone deacetylase inhibitor that exhibits broad spectrum antitumor activity in vitro and in vivo. Mol Cancer Ther 2008;7:1054-65.

45. Furumai R, Matsuyama A, Kobashi N, et al. FK228 (depsipeptide) as a natural prodrug that inhibits class I histone deacetylases. Cancer Res 2002;62:4916-21.

46. Miller TA, Witter DJ, Belvedere S. Histone deacetylase inhibitors. J Med Chem 2003; 46:5097-116.

47. Marks PA, Richon VM, Miller T, Kelly WK. Histone deacetylase inhibitors. Adv Cancer Res 2004;91:137-68.

48. Bhalla KN. Epigenetic and chromatin modifiers as targeted therapy of hematologic malignancies. J Clin Oncol 2005;23:3971-93.

49. Hu E, Dul E, Sung CM, et al. Identification of novel isoform-selective inhibitors within class I histone deacetylases. J Pharmacol Exp Ther 2003;307:720-8.

50. Deleu S, Fraczek J, Lukaszuk A, et al.
Screening of Trichostatin analogues based on cellular potency in the 5T33MM model. J Cancer Molecules 2008;4:117-21.

51. Fraczek J, Deleu S, Lukaszuk A, et al. Screening of amide analogues of Trichostatin A in cultures of primary rat hepatocytes: search for potent and safe HDAC inhibitors. Invest New Drugs 2008; Sep 30. [Epub ahead of print].

52. Urashima M, Ogata A, Chauhan D, et al. Interleukin- 6 promotes multiple myeloma cell growth via phosphorylation of retinoblastoma protein. Blood 1996;88:2219-27.

53. Finnin MS, Donigian JR, Cohen A, et al. Structures of a histone deacetylase homologue bound to the TSA and SAHA inhibitors. Nature 1999;401:188-93.

54. Van Ommeslaeghe K, Elaut G, Brecx V, et al. Amide analogues of TSA: synthesis, binding mode analysis and HDAC inhibition. Bioorg Med Chem Lett 2003;13:18614.

55. Mitsiades CS, Mitsiades NS, McMullan $\mathrm{CJ}$, et al. Transcriptional signature of histone deacetylase inhibition in multiple myeloma: biological and clinical implications. Proc Natl Acad Sci USA 2004;101:540-5.

56. Neri P, Tagliaferri P, Di Martino MT, et al. In vivo anti-myeloma activity and modulation of gene expression profile induced by valproic acid, a histone deacetylase inhibitor. Br J Haematol 2008;143:520-31.

57. Van Lint C, Emiliani S, Verdin E. The expression of a small fraction of cellular genes is changed in response to histone hyperacetylation. Gene Expr 1996;5:24553.

58. Gray SG, Qian CN, Furge K, et al. Microarray profiling of the effects of histone deacetylase inhibitors on gene expression in cancer cell lines. Int $\mathrm{J}$ Oncol 2004; 24:773-95.

59. Peart MJ, Smyth GK, van Laar RK, et al. Identification and functional significance of genes regulated by structurally different histone deacetylase inhibitors. Proc Natl Acad Sci USA 2005;102:3697-702.

60. Lavelle D, Chen YH, Hankewych M, DeSimone J. Histone deacetylase inhibitors increase p21(WAF1) and induce apoptosis of human myeloma cell lines independent of decreased IL-6 receptor expression. Am J Hematol 2001;68:170-8.

61. Kaiser M, Zavrski I, Sterz J, et al. The effects of the histone deacetylase inhibitor valproic acid on cell cycle, growth suppression and apoptosis in multiple myeloma. Haematologica 2006;91:248-51.

62. Gomez-Benito M, Martinez-Lorenzo MJ, Anel A, et al. Membrane expression of DR4, DR5 and caspase- 8 levels, but not Mcl-1, determine sensitivity of human myeloma cells to Apo2L/TRAIL. Exp Cell Res 2007;313:2378-88.

63. Schwartz C, Palissot V, Aouali N, et al. Valproic acid induces non-apoptotic cell death mechanisms in multiple myeloma cell lines. Int J Oncol 2007;30:573-82.

64. Mitsiades N, Mitsiades CS, Richardson PG, et al. Molecular sequelae of histone deacetylase inhibition in human malignant B cells. Blood 2003;101:4055-62.

65. Catley L, Weisberg E, Tai YT, et al. NVPLAQ824 is a potent novel histone deacetylase inhibitor with significant activity against multiple myeloma. Blood 2003;102: 2615-22.

66. Feng R, Ma H, Hassig CA, et al. KD5170, a novel mercaptoketone-based histone deacetylase inhibitor, exerts antimyeloma effects by DNA damage and mitochondrial signaling. Mol Cancer Ther 2008;7:1494-505.

67. Khan SB, Maududi T, Barton K, et al. Analysis of histone deacetylase inhibitor, depsipeptide (FR901228), effect on multiple myeloma. Br J Haematol 2004;125:15661.

68. Golay J, Cuppini L, Leoni F, et al. The histone deacetylase inhibitor ITF2357 has anti-leukemic activity in vitro and in vivo and inhibits IL-6 and VEGF production by stromal cells. Leukemia 2007;21:1892900.

69. Maiso P, Carvajal-Vergara X, Ocio EM, et al. The histone deacetylase inhibitor LBH589 is a potent antimyeloma agent that overcomes drug resistance. Cancer Res 2006; 66:5781-9.

70. Pellat-Deceunynk C, Amiot M, Bataille R, et al. Human myeloma cell lines as a tool for studying the biology of multiple myeloma: a reappraisal 18 years after. Blood 1995;86:4001-2.

71. Catley L, Weisberg E, Kiziltepe T, T et al. Aggresome induction by proteasome inhibitor bortezomib and alpha-tubulin hyperacetylation by tubulin deacetylase (TDAC) inhibitor LBH589 are synergistic in myeloma cells. Blood 2006;108:3441-9.

72. Deleu S, Lemaire M, Arts J, et al. The effect of JNJ-26481585, a novel hydroxamate-based histone deacetylase inhibitor, on the development of multiple myeloma in the 5T2MM and 5T33MM murine models. Leukemia 2009 in press.

73. Caers J, Asosingh K, Van Riet I, et al. Of mice and men: disease models of multiple myeloma. Drug Discovery Today: Disease Models 2004;1:373-80.

74. Vanderkerken K, Asosingh K, Croucher P, Van Camp B. Multiple myeloma biology: lessons from the 5TMM models. Immunol Rev 2003;194:196-206.

75. Ashkenazi A. Targeting death and decoy receptors of the tumour-necrosis factor 
superfamily. Nat Rev Cancer 2002;2:420 30 .

76. Oancea M, Mani A, Hussein MA, Almasan A. Apoptosis of multiple myeloma. Int $\mathrm{J}$ Hematol 2004;80:224-31.

77. Nakata S, Yoshida T, Horinaka M, et al. Histone deacetylase inhibitors upregulate death receptor 5/TRAIL-R2 and sensitize apoptosis induced by TRAIL/APO2-L in human malignant tumor cells. Oncogene 2004;23:6261-71.

78. van de Donk NW, Lokhorst HM, Bloem AC. Growth factors and antiapoptotic signaling pathways in multiple myeloma. Leukemia 2005;19:2177-85.

79. Green DR. Apoptotic pathways: paper wraps stone blunts scissors. Cell 2000;102: 1-4.

80. Burma S, Chen BP, Murphy M, et al. ATM phosphorylates histone $\mathrm{H} 2 \mathrm{AX}$ in response to DNA double-strand breaks. J Biol Chem 2001;276:42462-7.

81. Shao Y, Gao Z, Marks PA, Jiang X. Apoptotic and autophagic cell death induced by histone deacetylase inhibitors. Proc Natl Acad Sci USA 2004;101:18030-5.

82. Yamamoto S, Tanaka K, Sakimura R, et al. Suberoylanilide hydroxamic acid (SAHA) induces apoptosis or autophagy-associated cell death in chondrosarcoma cell lines. Anticancer Res 2008;28:1585-91.

83. Gozuacik D, Kimchi A. Autophagy as a cell death and tumor suppressor mechanism. Oncogene 2004;23:2891-906.

84. Ely S, Di Liberto M, Niesvizky R, et al. Mutually exclusive cyclin-dependent kinase 4/cyclin D1 and cyclin-dependent kinase 6/cyclin D2 pairing inactivates retinoblastoma protein and promotes cell cycle dysregulation in multiple myeloma. Cancer Res 2005;65:11345-53.

85. Dib A, Barlogie B, Shaughnessy JD Jr, Kuehl WM. Methylation and expression of the p16INK4A tumor suppressor gene in multiple myeloma. Blood 2007;109:13378.

86. Gonzalez-Paz N, Chng WJ, McClure RF, et al. Tumor suppressor p16 methylation in multiple myeloma: biological and clinical implications. Blood 2007;109:1228-32.

87. Wickner S, Maurizi MR, Gottesman S. Posttranslational quality control: folding, refolding, and degrading proteins. Science 1999;286:1888-93.

88. Bennett EJ, Bence NF, Jayakumar R, Kopito RR. Global impairment of the ubiquitin-proteasome system by nuclear or cytoplasmic protein aggregates precedes inclusion body formation. Mol Cell 2005; 17:351-65.

89. Kopito RR. Aggresomes, inclusion bodies and protein aggregation. Trends Cell Biol 2000;10:524-30.
90. Kawaguchi Y, Kovacs JJ, McLaurin A, et al. The deacetylase HDAC6 regulates aggresome formation and cell viability in response to misfolded protein stress. Cell 2003;115:727-38.

91. Nawrocki ST, Carew JS, Maclean KH, et al. Myc regulates aggresome formation, the induction of Noxa, and apoptosis in response to the combination of bortezomib and SAHA. Blood 2008;112:2917-26.

92. Hideshima T, Bradner JE, Wong J, et al. Small-molecule inhibition of proteasome and aggresome function induces synergistic antitumor activity in multiple myeloma. Proc Natl Acad Sci USA 2005;102:8567-72.

93. Deroanne CF, Bonjean K, Servotte S, et al. Histone deacetylases inhibitors as antiangiogenic agents altering vascular endothelial growth factor signaling. Oncogene 2002;21:427-36.

94. Michaelis M, Michaelis UR, Fleming I, et al. Valproic acid inhibits angiogenesis in vitro and in vivo. Mol Pharmacol 2004;65:520-7.

95. Qian DZ, Kato Y, Shabbeer S, et al. Targeting tumor angiogenesis with histone deacetylase inhibitors: the hydroxamic acid derivative LBH589. Clin Cancer Res 2006; 12:634-42.

96. Dong XF, Song Q, Li LZ, et al. Histone deacetylase inhibitor valproic acid inhibits proliferation and induces apoptosis in KM3 cells via downregulating VEGF receptor. Neuro Endocrinol Lett 2007;28:775-80.

97. Kitazoe K, Abe M, Hiasa M, et al. Valproic acid exerts anti-tumor as well as antiangiogenic effects on myeloma. Int $\mathrm{J}$ Hematol 2009;89:45-57.

98. De Bruyne E, Bos TJ, Asosingh K, et al. Epigenetic silencing of the tetraspanin CD9 during disease progression in multiple myeloma cells and correlation with survival. Clin Cancer Res 2008;14:291826.

99. Dmoszynska A. Diagnosis and the current trends in multiple myeloma therapy. Pol Arch Med Wewn 2008;118:563-6.

100. Adams J. The proteasome: structure, function, and role in the cell. Cancer Treat Rev 2003;29:3-9.

101. Hideshima T, Mitsiades C, Akiyama M, et al. Molecular mechanisms mediating antimyeloma activity of proteasome inhibitor PS-341. Blood 2003;101:1530-4.

102. Hideshima T, Richardson P, Chauhan D, et al. The proteasome inhibitor PS-341 inhibits growth, induces apoptosis, and overcomes drug resistance in human multiple myeloma cells. Cancer Res 2001;61: 3071-6.

103. Mitsiades N, Mitsiades CS, Poulaki V, et al. Molecular sequelae of proteasome inhibition in human multiple myeloma cells. Proc Natl Acad Sci USA 2002;99:14374-9.

104. Feng R, Oton A, Mapara MY, et al. The histone deacetylase inhibitor, PXD101, potentiates bortezomib-induced anti-multiple myeloma effect by induction of oxidative stress and DNA damage. $\mathrm{Br} \mathrm{J}$ Haematol 2007;139:385-97.

105. Fandy TE, Shankar S, Ross DD, et al. Interactive effects of HDAC inhibitors and TRAIL on apoptosis are associated with changes in mitochondrial functions and expressions of cell cycle regulatory genes in multiple myeloma. Neoplasia 2005;7:646-57.

106. Rosato RR, Grant S. Histone deacetylase inhibitors in clinical development. Expert Opin Investig Drugs 2004;13:21-38.

107. Kiziltepe T, Hideshima T, Catley L, et al. 5Azacytidine, a DNA methyltransferase inhibitor, induces ATR-mediated DNA double-strand break responses, apoptosis, and synergistic cytotoxicity with doxorubicin and bortezomib against multiple myeloma cells. Mol Cancer Ther 2007;6:1718-27.

108. Jones PA, Baylin SB. The fundamental role of epigenetic events in cancer. Nat Rev Genet 2002;3:415-28.

109. Ocio EM, Mateos MV, Maiso P, et al. New drugs in multiple myeloma: mechanisms of action and phase I/II clinical findings. Lancet Oncol 2008;9:1157-65.

110. Cameron EE, Bachman KE, Myohanen S, et al. Synergy of demethylation and histone deacetylase inhibition in the reexpression of genes silenced in cancer. Nat Genet 1999;21:103-7.

111. Keen JC, Yan L, Mack KM, et al. A novel histone deacetylase inhibitor, scriptaid, enhances expression of functional estrogen receptor alpha (ER) in ER negative human breast cancer cells in combination with 5-aza 2'-deoxycytidine. Breast Cancer Res Treat 2003;81:177-86.

112. Klisovic MI, Maghraby EA, Parthun MR, et al. Depsipeptide (FR 901228) promotes histone acetylation, gene transcription, apoptosis and its activity is enhanced by DNA methyltransferase inhibitors in AML1/ETO-positive leukemic cells. Leukemia 2003;17:350-8.

113. Shaker S, Bernstein M, Momparler LF, Momparler RL. Preclinical evaluation of antineoplastic activity of inhibitors of DNA methylation (5-aza-2'-deoxycytidine) and histone deacetylation (trichostatin A, depsipeptide) in combination against myeloid leukemic cells. Leuk Res 2003;27:437-44.

114. Zhu WG, Otterson GA. The interaction of histone deacetylase inhibitors and DNA methyltransferase inhibitors in the treat- 
ment of human cancer cells. Curr Med Chem Anticancer Agents 2003;3:187-99.

115. Du HL, Ren LM, Chen H, et al. Re-expression of p16 gene in the myeloma cell line U266 induced by synergy of sodium butyrate and 5-Aza-2'-deoxycytidine. Di Yi Jun Yi Da Xue Xue Bao 2002;22:981-4.

116. De Bruyne E, Bos T, Deleu S, et al. Regulation of Bim expression by IGF-1 in the 5T33MM murine model for multiple myeloma. ASH Annual Meeting Abstracts 2007;110:3512.

117. Atadja P, Shao W, Growney J, et al. LBH589: efficacy and protection of bone integrity in multiple myeloma as demonstrated in cell lines and in vivo mouse model. Haematologica 2007;92:151

118. Harrison SJ, Quach H, Yuen K, et al. High response rates with the combination of bortezomib, dexamethasone and the panhistone deacetylase inhibitor romidepsin in patients with relapsed or refractory multiple myeloma in a phase $\mathrm{I} / \mathrm{II}$ clinical trial. ASH Annual Meeting Abstracts 2008;112: 3698 .

119. Ottmann OG, Spencer A, Prince HM, et al. Phase IA/II study of oral panobinostat (LBH589), a novel pan-deacetylase inhibitor (DACi) demonstrating efficacy in patients with advanced Hematologic Malignancies. ASH Annual Meeting Abstracts 2008;112:958.

120.Wolf JL, Siegel D, Matous J, et al. A Phase II Study of Oral Panobinostat (LBH589) in Adult Patients with Advanced Refractory Multiple Myeloma. ASH Annual Meeting Abstracts 2008;112:2774.

121.Siegel Dd, Sezer 0, San Miguel JF, et al. A Phase IB, Multicenter, Open-Label, DoseEscalation Study of Oral Panobinostat (LBH589) and I.V. Bortezomib in Patients with Relapsed Multiple Myeloma. ASH Annual Meeting Abstracts 2008;112:2781.

122.Richardson P, Mitsiades C, Colson K, et al. Phase I trial of oral vorinostat (suberoylanilide hydroxamic acid, SAHA) in patients with advanced multiple myeloma. Leuk Lymphoma 2008;49:502-7.

123.Weber D, Badros AZ, Jagannath S, et al. Vorinostat Plus Bortezomib for the Treatment of Relapsed/Refractory Multiple Myeloma: Early Clinical Experience. ASH Annual Meeting Abstracts 2008;112:871.

124.Badros A, Philip S, Niesvizky R, et al. Phase I Trial of Suberoylanilide Hydroxamic Acid (SAHA) + Bortezomib (Bort) in Relapsed Multiple Myeloma (MM) Patients (pts). ASH Annual Meeting Abstracts 2007;110: 1168.

125.Galli M, Salmoiraghi S, Golay J, et al. A Phase II Multiple Dose Clinical Trial of Histone Deacetylase Inhibitor ITF2357 in Patients with Relapsed or Progressive Multiple Myeloma: Preliminary Results. ASH Annual Meeting Abstracts 2007;110: 1175. 